\title{
12 AÑOS DE EDUTEC-E REVISTA ELECTRÓNICA DE TECNOLOGÍA EDUCATIVA
}

\author{
Jesús Salinas Ibáñez \\ jesús.salinas@uib.es \\ Facultad de Ciencias de la Educación \\ Universidad de las Islas Baleares
}

\section{RESUMEN.}

El trabajo se ocupa de la evolución que ha experimentado la revista desde su creación en 1995. Se describen los antecedentes y momentos iniciales, se analiza el impacto e influencia que ha tenido en el campo de la Tecnología educativa. Se revisa la línea editorial que se ha seguido y se ofrecen elementos de reflexión sobre el papel que dicha revista ha tenido en la evolución y desarrollo de las TIC en la educación en el ámbito de habla hispana.

PALABRAS CLAVE: publicación electrónica, tecnología educativa

\begin{abstract}
.
The paper takes care of the evolution that has experienced the Edutec-e review from its creation in 1995. The antecedents and initial moments are described, it analyzes the impact and influence that it has had in the field of the Educational Technology. The publishing line that it has been followed is reviewed and reflection elements are offered on the rol that this review has had in the evolution and development of the TIC in education in the Spanish world.
\end{abstract}

KEY WORD: electronic review, educational technology 


\section{Los antecedentes}

Corre el año 1995 y se celebra los días 22, 23 y 24 de Noviembre EDUTEC'05 el II Congreso de Nuevas Tecnologías de la Información y la Comunicación para la Educación. Como una de las actividades previas de dicho congreso, el Comité Científico acuerda poner en marcha una experiencia de debate previo sobre una de las ponencias a presentar en el Congreso. En concreto la que presenta el Dr. Adalberto Ferrández: "El formador en el espacio formativo de las redes"(Ferrández, 1996). La experiencia se desarrolla mediante una lista de discusión organizada a tal fin con los miembros del Comité Científico y que denominamos edutec-l. Allí la propuesta provisional fue sometida a debate y los miembros de dicha lista aportaron ideas, alternativas, experiencias, críticas, etc., a la propuesta del Dr. Fernández que le sirvieron a éste para cerrar su ponencia definitiva.

A raíz de dicha experiencia se propone en las conclusiones del Congreso continuar y ampliar la actividad del la lista, abriéndola a todos los asistentes y a los interesados en la temática en el ámbito académico. Así mismo, se decide poner en marcha una revista electrónica que sirva de foro de reflexión para el grupo de profesores y congresistas interesados.

Ese mismo mes de noviembre se ponen ambos proyectos en marcha con una lista de 76 profesores -aquellos asistentes que dejaron su correo electrónico en el congreso- y con el núm. 0 de EDUTEC-E Revista Electrónica de Tecnología Educativa. En este primer número se publica el trabajo "Satélites, cable, redes: Un nuevo panorama para la producción de televisión educativa" de mi autoría y que como nota nostálgicohistórica se reproduce en este mismo número.

En sus orígenes, por lo tanto, dicha revista se distribuye por la lista de discusión edutec-l, incluye un sólo artículo y su objetivo es dinamizar debate de ideas sobre temas que a la comunidad recién generada estaba interesando. Puede ser clarificador el este sentido lo que se dice en la presentación de la misma:

"La puesta en marcha de la EDUTEC-E. Revista Electrónica de Tecnología Educativa quiere ser uno de los frutos del reciente II Congreso de Nuevas Tecnologías de la Información y la Comunicación para la Educación celebrado los pasados días 22, 23 y 24 de noviembre en Palma de Mallorca.

A través de este canal se quiere ofrecer, más que materiales de difusión similares a los materiales impresos, propuestas para el debate y la reflexión que posteriormente pueden publicarse en Pixel-Bit. Revista de Medios de Educación o en otras revistas del campo de la Tecnología Educativa. 
Esperamos que EDUTEC-E. Revista Electrónica de Tecnología Educativa se convierta en foro de debate y reflexión sobre los temas candentes del campo de la Tecnología Educativa.

Empezamos la andadura de esta revista con un trabajo que supone el marco de reflexión de EEOS como asociación, pero que puede servir de elemento de discusión entre todos nosotros".

Para hacerse una idea de la actividad del colectivo $y$, en consecuencia, de la distribución de la revista, puede servir el hecho de que en el año 1999 ya se cuenta con más de 700 suscriptores y en marzo de 2001 contaba ya con 933. En los momentos con más suscriptores se ha sobrepasado los 1.100.

La distribución geográfica también podemos considerarla, siempre en proporción al número de suscritores, constante (entre 19 y 23 países representados). Como muestra representativa podemos ver la distribución por países de marzo de 2001, que puede considerarse el ecuador de esta andadura (Cuadro 1).

En el año 2000 se produce un cambio de formato. En esta nueva etapa la revista recoge artículos y trabajos de reflexión generados desde el ámbito de la Tecnología Educativa y el principal objetivo es no solo difundir trabajos y experiencias de nuestro entorno profesional sino servir de plataforma para el diálogo, el intercambio de ideas y la participación.

De hecho, la Revista Electrónica de Tecnología Educativa forma parte ya del proyecto más amplio: Edutec. Comunidad Virtual de Tecnología Educativa que integra: Edutec-I (foro de discusión y intercambio profesional), Edutec-e Revista Electrónica de Tecnología Educativa, un espacio compartido basado en el BSCW para grupos de trabajo interuniversitario, espacio de chat... además de otros servicios de documentación e información. La Comunidad Virtual de Tecnología Educativa es promovida y diseñada por EDUTEC. Asociación para el Desarrollo de la Tecnología Educativa y las Nuevas Tecnologías aplicadas a la Educación, que encarga su gestión, organización y mantenimiento al Grupo de Tecnología Educativa de la Universitat de les Illes Balears.

Edutec-e. Revista Electrónica de Tecnología Educativa, a partir de este momento se publica exclusivamente en formato web, se independiza de alguna manera de la lista edutec-I y adquiere periodicidad trimestral constando de las siguientes secciones: 


\begin{tabular}{|l|c|}
\hline \multicolumn{1}{|c|}{ País } & Núm. Suscriptores \\
\hline * Argentina & 96 \\
$*$ Australia & 1 \\
$*$ Bolivia & 1 \\
$*$ Brazil & 78 \\
$*$ Chile & 27 \\
$*$ Colombia & 12 \\
$*$ Costa Rica & 1 \\
$*$ Cuba & 13 \\
$*$ France & 6 \\
$*$ Germany & 1 \\
$*$ Italy & 1 \\
$*$ Mexico & 10 \\
$*$ Niue & 1 \\
$*$ Panama & 2 \\
$*$ Peru & 7 \\
$*$ Portugal & 3 \\
$*$ Soviet Union & 1 \\
$*$ Spain & 408 \\
$*$ USA & 251 \\
$*$ Uruguay & 5 \\
$*$ Venezuela & 7 \\
(Nótese que son adjudicados a USA & \\
todos los .com, etc) & \\
\hline Total USUARIOS suscritos a la lista & 21 \\
\hline Total países representados & \\
\hline
\end{tabular}

Cuadro 1: Suscriptores de edutec-I por países (marzo 2001). Fuente: listserv de Rediris

Artículos. Publicación de artículos, en forma de trabajos de investigación o experiencias

Opinión. Publicación de artículos de opinión, resultados de trabajos, etc. Con el principal objetivo de potenciar el diálogo y la participación dentro de la lista de distribución Edutec.

Sumarios. Semanalmente se ofrecerá un sumario de las principales líneas de debate, así como de las aportaciones y conclusiones generadas.

Estadísticas. Junto con cada sumario se presentaran los datos en cifras sobre la participación para cada una de las líneas temáticas iniciadas

Desde el año 2000 Edutec-e se integra como un proyecto más de la Comunidad Virtual de Tecnología Educativa, lo mismo que ocurre con edutec-I, la lista de discusión. 


\section{Edutec-e y la Comunidad Virtual de Tecnología Educativa}

Un hito importante en la marcha de la revista lo constituye la inauguración del sitio de la Comunidad Virtual de Tecnología Educativa (25-9-98), en el marco de un proyecto de REDIRIS que en aquel momento apoyaba iniciativas de grupos interuniversitarios de carácter académico. Uno de los elementos centrales de la comunidad lo constituye Edutec-e y a partir de ese momento los sucesivos números, además de distribuirlos por la lista, son publicados en formato web en dicha Comunidad Virtual.

Como señalan Ordinas, Pérez y Salinas (1999), la Comunidad Virtual de Tecnología Educativa supone la evolución de una comunidad ya existente, congregada alrededor de la lista de distribución Edutec-l, ampliando sus canales y posibilidades comunicativas, añadiendo posibilidad de compartir documentación y recursos, de teleinvestigación, de trabajo colaborativo, etc.

En efecto, la revista electrónica encaja perfectamente en el proyecto de la Comunidad Virtual de Tecnología Educativa, al pretender ésta servir de plataforma para potenciar el conocimiento y el uso de las nuevas tecnologías en el ámbito educativo mediante la distribución de materiales periódicos relacionados con la temática, proporcionar un canal de difusión de actividades, experiencias relacionadas y la puesta a disposición del colectivo de recursos educativos.

La Comunidad Virtual de Tecnología Educativa pretende servir de plataforma para potenciar el conocimiento y el uso de las nuevas tecnologías en el ámbito educativo mediante la distribución de materiales periódicos relacionados con la temática, proporcionar un canal de difusión de actividades, experiencias relacionadas y la puesta a disposición del colectivo de recursos educativos (Ordinas, Pérez y Salinas, 1999).

Concretamente pretende ser un espacio donde los profesionales de este ámbito compartamos, intercambiemos y promovamos proyectos relacionados con la explotación de las posibilidades educativas de las tecnologías de la comunicación, mediante:

- EL debate académico en el ámbito iberoamericano respecto a las tecnologías de la comunicación aplicadas a la educación.

- El intercambio de experiencias referidas al diseño, producción, uso y evaluación de nuevos medios didácticos.

- La organización de debates telemáticos, y otras actividades apoyadas en las posibilidades comunicativas de las redes. 
- La experimentación de herramientas de aprendizaje colaborativo

- Experimentación y evaluación de Webtools, etc...

- Promover proyectos de innovación por parte de grupos de profesores del colectivo, etc...

\section{La producción científica}

Hasta julio de 2007 se han publicado 81 artículos en los 23 números que han aparecido de la revista (Del 0 al 10 en la primera etapa en la que se publicada un solo artículo por número, y el resto en la segunda).

Algunos de estos artículos podemos considerar que han tenido gran impacto en la comunidad que se dedica a la Tecnología Educativa. Como puede comprobarse en el Cuadro 2, se han publicado varios trabajos que han tenido enorme repercusión en nuestro ámbito. Ofrecemos en dicho cuadro, sin ánimo de ser exhaustivos, ni habiendo utilizado sistemas rigurosos para la obtención de la información, algunos de los trabajos con mayor presencia en Internet.

\begin{tabular}{|c|c|}
\hline Artículo & $\begin{array}{l}\text { Núm. } \\
\text { resultados }\end{array}$ \\
\hline $\begin{array}{l}\text { - B. DE BENITO, Herramientas para la creación, distribución y gestión de } \\
\text { cursos a través de Internet Edutec № } 12,06 / 00\end{array}$ & 428.000 \\
\hline $\begin{array}{l}\text { - F. NEGRE, Reflexión sobre posibles razones de la dificultad de } \\
\text { introducir las nuevas tecnologías en el campo de la educación especial } \\
\text { Edutec № 9, 10/98 }\end{array}$ & 388.000 \\
\hline $\begin{array}{l}\text { - G. CAPLAN, Un libro sin papel. Capítulo } 1 \text { de Editar sin papel. Editorial } \\
\text { JVE Siglo XXI/Informática2 Edutec № 12, 06/00 }\end{array}$ & 259.000 \\
\hline - A. DE LA TORRE, Web Educativa 2.0. Edutec № 20, 01/06 & 235.000 \\
\hline $\begin{array}{l}\text { - J. SALINAS, Enseñanza flexible, aprendizaje flexible, aprendizaje } \\
\text { abierto. Las redes como herramienta para la formación Edutec № 10, } \\
\text { 02/99 }\end{array}$ & 63.300 \\
\hline $\begin{array}{l}\text { - R. RODRÍGUEZ LAMAS, La informática educativa en el contexto actual. } \\
\text { Edutec № } 13,11 / 00\end{array}$ & 49.900 \\
\hline $\begin{array}{l}\text { - J. ADELL, Tendencias en Educación en la Sociedad de las Tecnologías de } \\
\text { la Información. Edutec № 7, 11/97 }\end{array}$ & 36.800 \\
\hline - J. CABERO, Nuevas Tecnologías, Comunicación y Educación. Edutec № & 36.600 \\
\hline
\end{tabular}




\begin{tabular}{|l|c|}
\hline \multicolumn{1}{|l|}{ 1, 02/96 } & \\
\hline - $\begin{array}{l}\text { S. URBINA, Algunas consideraciones en torno al sotware para } \\
\text { Educación Infantil. Edutec № 13, 11/00 }\end{array}$ & 32.000 \\
\hline - $\quad \begin{array}{l}\text { J. CABERO, Comunidades virtuales para el aprendizaje. Su utilización en } \\
\text { la enseñanza Edutec № 20, 01/06 }\end{array}$ & 30.300 \\
\hline $\begin{array}{l}\text { * Para la búsqueda realizada se han utilizado el nombre del autor más los tres primeros sustantivos del título del artículo y se ha } \\
\text { utilizado Google como buscador (11-10-07) }\end{array}$ \\
\hline
\end{tabular}

Cuadro 2.- Resultados obtenidos en buscador Google de 10 artículos más frecuentes publicados en Edutec-e

La validez del factor de impacto como indicador de visibilidad es un hecho ampliamente aceptado. A pesar de contar con herramientas de mayor capacidad y muy variadas prestaciones de cara a la evaluación del mismo, el incremento y los nuevos tipos de público y de interacciones complican la evaluación de la calidad científica de las revistas electrónicas como la nuestra. Pero sin querer entrar en el debate sobre la medida de dicho impacto de la producción científica y en especial de las publicaciones periódicas en formato electrónico y su consideración por los sistemas de medida de los mismos - el factor de impacto experimenta importantes variaciones según las disciplinas consideradas, etc-, queremos ofrecer en el Cuadro 3, las visitas que hemos podido contabilizar en un periodo concreto de tiempo (los 12 meses que van de marzo 2001 a febrero 2002) por algunos de los artículos más frecuentados del periodo. No se trata de indicador de dicho factor de impacto, pero lo traemos aquí solamente como un ejemplo, conscientes de que introducimos sesgos (cercanía de la fecha de publicación, si se ha publicado en medio del mismo, etc..) que deben llevarnos a considerar dichos datos con las precauciones debidas (mayor probabilidad de presencia de artículos publicados recientemente, etc.).

Se ofrecen así mismo los datos globales de la Comunidad Virtual de Tecnología educativa que dan una idea del peso que la revista tiene en el proyecto.

\begin{tabular}{|c|c|}
\hline Artículo & $\begin{array}{l}\text { Visitas } \\
\text { 03-01 a 02-02 }\end{array}$ \\
\hline $\begin{array}{l}\text { - B. DE BENITO, Herramientas para la creación, distribución y gestión de } \\
\text { cursos a través de Internet Edutec № 12, 06/00 }\end{array}$ & 10.065 \\
\hline $\begin{array}{l}\text { - S. URBINA, Algunas consideraciones en torno al software para Educación } \\
\text { Infantil. Edutec № } 13,11 / 00 \text {. }\end{array}$ & 7.517 \\
\hline $\begin{array}{l}\text { - R. RODRÍGUEZ LAMAS, La informática educativa en el contexto actual. } \\
\text { Edutec № } 13,11 / 00\end{array}$ & 6.792 \\
\hline
\end{tabular}

- S. ALAVA, Información, saber y ciberespacio: un desafío para la autoformación. Edutec № 11, 02/00

- B. GROS, La dimensión socioeducativa de los videojuegos. Edutec № 12, $06 / 00$ 


\begin{tabular}{|c|c|c|c|}
\hline$\bullet$ & $\begin{array}{l}\text { G. CAPLAN, Un libro sin pa } \\
\text { Siglo XXI/Informática2 Edu }\end{array}$ & $\begin{array}{l}\text { tulo } 1 \text { de Editar sin papel. Editorial JVE } \\
2,06 / 00\end{array}$ & 3.343 \\
\hline & $\begin{array}{l}\text { COOKSON, P.S. La Práctica } \\
\text { la Universidad de Athabas } \\
14,05 / 01\end{array}$ & $\begin{array}{l}\text { ación Superior a Distancia: El Ejemplo de } \\
\text { iversidad Abierta en Canadá Edutec № }\end{array}$ & 3.018 \\
\hline & $\begin{array}{l}\text { M. ESPINOSA, Estrategias c } \\
\text { participación y construcció } \\
\text { electrónicos. (publicado er } \\
\text { núm. 2) Edutec № } 11,02 /(\end{array}$ & $\begin{array}{l}\text { ración como mecanismo de } \\
\text { hocimiento en grupos de discusión } \\
\text { textos. Campus de Monterrey, año 1, }\end{array}$ & 2.794 \\
\hline & $\begin{array}{l}\text { GARCíA ARETIO, L. La inno } \\
\text { impreso a la tecnología UN }\end{array}$ & $\begin{array}{l}\text { ermanente en la UNED: del material } \\
\text { tec № } 14,05 / 01\end{array}$ & 2.739 \\
\hline & $\begin{array}{l}\text { SHARON G. SOLLOWAY, ED } \\
\text { de comunidades on-line. L } \\
\text { I@s alumn@s en el ciberes }\end{array}$ & $\begin{array}{l}\text { HARRIS y GRACE H. MAYER Creación } \\
\text { ación de las necesidades y los deseos de } \\
\text { dutec № } 11,02 / 00\end{array}$ & 765 \\
\hline \multicolumn{4}{|c|}{ TOTALES DEL PERIODO (CVU Tecnología Educativa) } \\
\hline \multicolumn{4}{|c|}{ Accesos } \\
\hline \multicolumn{4}{|c|}{ Archivos } \\
\hline \multicolumn{4}{|c|}{16.843} \\
\hline \multicolumn{4}{|c|}{ Visitas } \\
\hline \multicolumn{4}{|c|}{1.338 .332} \\
\hline \multicolumn{4}{|c|}{ Clientes } \\
\hline \multicolumn{4}{|c|}{ URLS } \\
\hline
\end{tabular}

Cuadro 3.- Accesos a diversos artículos de Edutec-e. Revista Electrónica de Tecnología Educativa durante el periodo 03-01 a 02-02 (ambos inclusive).

\section{La línea editorial}

Hay que decir que la línea editorial de la revista se ha mantenido a grandes rasgos tal y como se ideó en sus primeros momentos y que hemos descrito en el primer punto. Es indudable que las temáticas de los materiales producidos han ido evolucionando conforme ha evolucionado la tecnología y el pensamiento pedagógico respecto a su incorporación a los procesos de enseñanza-aprendizaje.

En términos generales, se puede decir que se sigue pretendiendo promover la cooperación respecto a la producción, la difusión, el uso y la evaluación de materiales y programas educativos, divulgar la experimentación e investigación en el campo de la aplicación de las nuevas tecnologías a la educación, y difundir experiencias para aumentar y mejorar la implantación de la Tecnologìa Educativa en los sistemas de enseñanza.

La política editorial de Edutec-e viene marcada por tres aspectos: 
a) La importancia de la publicación electrónica en un campo que se ocupa precisamente de las posibilidades que para la comunicación educativa aportan las TIC.

Quizá sea conveniente en este punto atender a la situación que nuestro campo presentaba en el momento del nacimiento de la revista para entender algunas cosas. Se trata de un momento en el que estas tecnologías están sufriendo profundos cambios que afectan a la enseñanza. Desde la perspectiva del usuario de formación, esta evolución ofrece en ese momento una doble tendencia aparentemente contradictoria, pero que en el fondo resulta complementaria: Por una parte, las crecientes posibilidades ofrecidas, sobretodo, por la evolución de los satélites de telecomunicaciones muestran una clara tendencia a audiencias potenciales extensas, hacia los mass-media entendidos como un mismo mensaje para grandes audiencias. Desde esta perspectiva, satisfacer las necesidades educativas de un conjunto tan heterogéneo de ciudadanos supone un desafío para las tecnologías aplicadas a la educación, pero también un fuerte compromiso con proporcionar acceso a la misma. Por otra parte, el desarrollo de nuevas y más asequibles herramientas microinformáticas ha potenciado otra tendencia hacia los self-media, hacia los medios de formación bajo control del usuario. Esto queda patente, sobre todo, en la evolución de los sistemas multimedia.

La evolución posterior es conocida, y como respuesta a esta situación, las telecomunicaciones presentan crecientes posibilidades. Pero en esta evolución, su integración en los procesos de enseñanza-aprendizaje debe atender, sin embargo, a múltiples factores. Entre ellos, uno de los más importantes es la disponilidad tecnológica. Es indudable que las instituciones de educación superior debían disponer de las últimas ventajas que proporcionaban las telecomunicaciones. Y aunque ello, no debía suponer esperar la 'buena tecnología', sino poner en marcha proyectos utilizando la tecnología disponible en cada momento, todo ello fue resultando de forma desigual.

En todo este periodo y desde una perspectiva realista, son importantes los estudios sobre la tecnología (tipo de tecnología a emplear, distancia y otros aspectos geográficos, existencia y disponibilidad de la tecnología, etc...), pero sobre todo con los aspectos relacionados con el currículum, la formación del personal, el diseño y producción del material, etc... Junto a estos estudios, en las universidades y salvo excepciones, se ve como necesaria una mayor investigación tanto en lo referente a los componentes tecnológicos, como a los didácticos, y un mayor número de experiencias cooperativas de producción y de investigación. 
b) La consideración de la publicación digital de libre acceso.

Pienso que esta característica, que por obvia a veces no es suficientemente considerada es una de las marcas características, que a mi entender toma clara importancia desde la perspectiva histórica que estamos realizando. Edutec-e se encuentra entre los proyectos editoriales pensados exclusivamente para la red, como proyectos diseñados específicamente para distribución por correo electrónico o navegando en Internet, y se encuentra entre aquellas que se distribuyen gratuitamente, siendo la única condición necesaria para acceder a su contenido el disponer de una conexión a la red y el software de navegación. Como la mayor parte de las revistas electrónicas de este tipo -a diferencia de las comerciales que pasan a publicarse también en formato digital, o de comerciales nacidas ya en este formato- han podido nacer gracias a Internet, que permite a cualquier persona la posibilidad de comunicarse con una audiencia potencial de millones de personas, cosa que en el mundo de la imprenta y del documento impreso sólo se podía conseguir con unas fuertes inversiones. Esto que puede parecer casi una perogrullada en la actualidad, no era tan sencillo en 1995.

La idea de base de nuestra revista radica en que los auténticos productores del conocimiento científico, los investigadores, no pierden el control sobre sus trabajos, sino que lo aportan a la comunidad científica de forma desinteresada, poniendo a la libre disposición de los lectores el material publicado. De hecho la responsabilidad del sitio web donde se alberga la revista y de todo el proyecto que lo arropa, recae e EDUTEC, Asociación para el Desarrollo de la Tecnología Educativa y Nuevas tecnologías a la Educación (organización sin ánimo de lucro entre cuyos fines se encuentra precisamente promover este tipo de proyectos).

c) La innovación en cuanto a la comunicación científica y otro tipo de información de interés para el campo.

Desde el nacimiento de la revista el sistema de divulgación del conocimiento científico ha ido experimentando profundos cambios, debido sobre todo al auge del canal privilegiado de difusión: Internet. Las actuales necesidades de divulgación de la información y el conocimiento científico encuentran en algunas de las características que las TIC presentan (accesibilidad, inmediatez, navegabilidad, internacionalización, economía, etc.) cumplida respuesta, sobre todo en campos como el nuestro, dedicado a las posibilidades que estas mismas tecnologías presentan para su aplicación en la mejora de los procesos educativos. 
En esta evolución, encontramos que junto al avance en la riqueza de contenidos, han aparecido nuevas posibilidades en la navegación que permiten un abanico de posibilidades de formatos de edición, archivo e intercambio (navegación cada vez más multimedia, estadísticas instantáneas de uso, recuperación de enlaces o avisos automáticos, las votaciones y sugerencias de los lectores, RSS y otros sistemas de sindicación, etc..).

En cualquier caso, las enormes posibilidades que la distribución, intercambio y recuperación de la información está experimentando, obliga a reconsiderar los formatos de distribución de la comunicación científica, o al menos a reflexionar sobre el sitio y función de este tipo de publicaciones digitales.

Teniendo todo ello en cuenta, podemos considerar que desde la revista, con la orientación del Consejo de Redacción, se ha procurado satisfacer las funciones que Smith (1999) atribuye a las revistas digitales. Entre las funciones explícitas: selección editorial de los materiales en función de la orientación temática en el estilo de la revista y/o según el posible interés para sus lectores; control de la calidad de los contenidos recibidos por el equipo editorial o los revisores que colaboran en la edición, control de la calidad de redacción y representación en los textos e imágenes; reconocimiento al trabajo de los investigadores por la publicación; consecución de reputación ante los lectores o bibliotecarios para las mismas cabeceras científicas merced a su autoridad, rigor, antigüedad, etc.; difusión o divulgación de información científica.

Pero sobre todo entendemos que se ha participado en cumplir lo que este mismo autor señala como funciones ocultas o subyacentes: definición de las áreas de la disciplina (mediante editoriales, o a través de la selección y revisión de documentos); definición de la comunidad de los lectores (función más evidente en el caso de las nuevas disciplinas o de las áreas emergentes); y archivo documental de la especialidad, (indirectamente, a través del archivo documental en bibliotecas virtuales y centros de recursos).

\section{Algunas reflexiones}

La ocasión de revisar lo realizado y analizar el panorama en el que se integra Edutec-e me ha llevado a reflexionar sobre algunos aspectos que rodean la publicación digital en general, pero que afectan al recorrido de nuestra revista y sobre el papel que en la comunidad académica ha tenido el proyecto: 
- Lo primero que llama la atención es el tema del impacto de la revista. La dificultad que entraña diferenciar el impacto medido y el verdadero impacto de los trabajos en la comunidad académica. La impresión, enormemente subjetiva por supuesto, de que algunos de los trabajos publicados en Edutec-e han tenido una gran difusión, han sido considerados por la comunidad académica del campo, han supuesto y suponen todavía en la actualidad trabajos de referencia en investigaciones y experiencias, choca con lo que en la comunidad científica hace referencia a impacto medido y a Índices de impacto. Probablemente, esto sea debido en parte a aspectos de los que nos ocupamos en puntos sucesivos. Y todo ello sin querer entrar en los temas de impacto medido según los sistemas al uso, etc., pero que presenta paradojas como que algunos trabajos de esta revista, que se pueden encontrar reproducidos en cientos $-y$ no es retórico- de sitios web, referenciados en innumerables ocasiones, con decenas de miles de accesos como puede ser el caso del trabajo de De Benito (2000) y unos cuantos más, no aparezcan en ninguno de los índices de impacto normalizados de la publicación científica española.

- Un elemento de reflexión, que se da también en otros ámbitos relacionados con el uso de las TIC es el traslado de las normas de publicación habituales en papel -que es lo que se hizo en el primer momento-, y que aparece como insuficiente en los entornos digitales (ausencia de enlaces a los autores, periodicidad, versiones en formatos alternativos, etc.). En otras palabras, en el contexto de la Sociedad de la Información y el Conocimiento, con enormes posibilidades de distribución, el formato sigue respondiendo a un tipo de publicación académica típica.

- Siempre es crítico el equilibrio entre el mantenimiento de la tradición y la flexibilidad para adaptarse a las situaciones cambiantes. En este caso la tradición, el mantenimiento de la revista reconocible a lo largo de 12 años, parece que ha ido adaptándose a los nuevos requerimientos. Si en la temática, y en el cumplimiento de algunas de las funciones que señala Smith (1999), se ha producido una gran evolución, aparecen aspectos negativos que provienen de aspectos formales de la web donde se alberga la revista, y que contribuye a una limitada visibilidad, por lo que el acceso a los contenidos, tanto de los usuarios como de los motores de búsqueda, se presenta como un aspecto a mejorar. Todo ello, teniendo en cuenta que algunas políticas en este sentido, desdibujan las fronteras de la edición académica tras políticas de visibilidad ante los buscadores o la diversidad de navegantes, lo que puede redundar en pérdida de reputación científica.

- EL papel de la revista en el conjunto de sistemas de distribución de la información científica en el campo de la Tecnología Educativa también debe suponer un elemento de reflexión. No es este el lugar para hablar de futuro, 
pero es importante señalar que en los últimos años han ido surgiendo publicaciones periódicas y otros sistemas de difusión de investigación, innovación y experiencias que hacen más visible el trabajo que se desarrolla en nuestro campo. Desde la perspectiva histórica, que es la que aquí nos ocupa, observamos algunas superposiciones e interferencias entre diversos proyectos -no parece pertinente hablar de competencia-, pero lo que parece más llamativo es que algunos de estos proyectos se presentan como si nada antes hubiera existido. Pienso que puede ser interesante un mapa de todos los proyectos que se ocupan de la implantación de las TIC en el sistema educativo.

- La deslocalización de los contenidos contribuye a la perdida de referencia y a bajar el impacto de la revista. La publicación de los materiales de nuestra revista en otros sitios web ha sido habitual a lo largo de estos años. En algunos casos, incluyendo la referencia completa, pero en otros muchos con deficientes referencias o habiendo desprovisto a los artículos de las mismas. En algunos casos, muchos de nosotros practicamos esta política de "deslocalizar" los materiales en lugar de referenciarlos adecuadamente, contribuyendo así a empobrecer el acceso del usuario a la información y no aportando impacto y visibilidad de la revista.

Al margen de estos y otros elementos de reflexión que podemos encontrar, se ha de valorar el papel que en la comunidad académica relacionada con el uso y desarrollo de la Tecnología Educativa ha tenido Edutec-e. Como se ha dicho, la persistencia de la revista reconocible a lo largo de 12 años, con la necesaria evolución experimentada quizá no la suficiente- supone ya un hito importante en este ámbito, donde proliferan proyectos, seminarios, congresos sobre TIC y aspectos concretos relacionados directa o indirectamente con la educación, que en muchos casos tienen vida efímera, fruto en ocasiones del oportunismo.

La influencia que en el seno del proyecto que supone la Comunidad Virtual de tecnología Educativa, ha experimentado la revista, su trayectoria y la perspectiva que ha mantenido siempre se proyecto colectivo y compartido, la convierte en uno de los proyectos más consolidados de habla hispana relacionado con las TIC y la educación.

Al final, este proyecto que se inició en EDUTEC'95 en Palma, pero que tiene su origen en aquel I Congreso de Nuevas Tecnologías de la Información y la Comunicación para la Educación de diciembre de 2003 en Badajoz, ha supuesto valiosas aportaciones a este campo, y, es seguro, que con el concurso de investigadores y académicos que sienten como suyo el proyecto, lo seguirá haciendo en el futuro. 


\section{Referencias}

- DE BENITO, B. (2000): Herramientas para la creación, distribución y gestión de cursos a través de Internet. Edutec, Revista Electrónica de Tecnología Educativa. N.12

- FERRÁNDEZ, A. (1996)."El formador en el espacio educativo de las redes". En Salinas,J. y otros (Coord): Redes de comunicación, redes de aprendizaje. Publicaciones de la Universidad de las Islas Baleares, Palma de Mallorca

- ORDINAS,C., PÉREZ GARCíAS, A. Y SALINAS, J. (1999): Comunidad virtual de Tecnología Educativa. Edutec. En Cabero,J. Y otros: Nuevas Tecnologías en la formación flexible y a distancia. Universidad de Sevilla. Sevilla.

- SMITH (1999). JOHN W. T. SMITH, "The Deconstructed Journal -a new model for Academic Publishing", Learned Publishing, Vol. 12, No. 2, Abril 1999. $<$ http://library.kent.ac.uk/library/papers/jwts/d-journal.htm>

\section{Para citar este artículo:}

SALINAS, Jesús (2008). «12 Años de EDUTEC-E. Revista Electrónica de Tecnología Educativa» [artículo en línea]. EDUTEC, Revista Electrónica de Tecnología Educativa. Núm. 25/Marzo 2008. [Fecha de consulta: $\mathrm{dd} / \mathrm{mm} / \mathrm{aa}$ ]. http://edutec.rediris.es/Revelec2/Revelec25/Edutec25 12 anos edutece revista electronica tecnologia educativa.html ISSN 1135-9250. 